\title{
Functional Magnetic Resonance Imaging Brain Mapping in Psychiatry: Methodological Issues Illustrated in a Study of Working Memory in Schizophrenia
}

Joseph H. Callicott, M.D., Nicolas F. Ramsey, Ph.D., Kathleen Tallent, B.A., Alessandro Bertolino, M.D., Michael B. Knable, M.D., Richard Coppola, Ph.D., Terry Goldberg, Ph.D., Peter van Gelderen, Ph.D., Venkata S. Mattay, M.D., Joseph A. Frank, M.D., Chrit T.W. Moonen, Ph.D., and Daniel R. Weinberger, M.D.

Functional magnetic resonance imaging (fMRI) is a potential paradigm shift in psychiatric neuroimaging. The technique provides individual, rather than group-averaged, functional neuroimaging data, but subtle methodological confounds represent unique challenges for psychiatric research. As an exemplar of the unique potential and problems of $f M R I$, we present a study of 10 inpatients with schizophrenia and 10 controls performing a novel " $n$ back" working memory (WM) task. We emphasize two key design steps: (1) the use of an internal activation standard (i.e., a physiological control region) to address activation validity, ard (2) the assessment of signal stability to control for "activation" artifacts arising from unequal signal variance across groups. In the initial analysis, all but one of the patients failed to activate dorsolateral prefrontal cortex (DLPFC) during the working memory task. However, some patients (and one control) also tended to show sparse control region activation in spite of normal motor performance, a result that raises doubts about the validity of the initial analysis and concerns about unequal subject motion. Subjects were then matched for signal variance (voxel stability), producing a subset of six patients and six controls. In this comparison, the internal activation standard (i.e., motor activation) was similar in both groups, and five of six patients, including two whom were neuroleptic-naive, failed to activate DLPFC. In addition, a tendency for overactivation of parietal cortex was seen. These results illustrate some of the promise and pitfalls of fMRI. Although fMRI generates individual brain maps, a specialized survey of the data is necessary to avoid spurious or unreliable findings, related to artifacts such as motion, which are likely to be frequent in psychiatric patients. [Neuropsychopharmacology 18:186-196, 1998] Published by Elsevier Science Inc.
From the Clinical Brain Disorders Branch (JHC, KT, AB, MBK, RC, TG, VSM, DRW), IRP, NIMH, NIH, Washington, DC; In vivo NMR Center (PVG, CTWM), BEIP, NCRR, NIH, Bethesda, Maryland; Laboratory of Diagnostic Radiology Research (JAF), OIR, NIH, Bethesda, Maryland; Department of Psychictry (NFR), University of Utrecht, Utrecht, The Netherlands.

Address correspondence to: Joseph H. Callicott, M.D., Clinical Brain Disorders Branch, Intramural Research Program, National Institute of Mental Health/National Institutes of Health, Building 10, Room 4N-317, Bethesda, MD 20892.

Received December 26, 1996; revised April 16, 1997; accepted June 4, 1997
KEY WORDS: fMRI; Schizophrenia; Prefrontal; Memory; Cognition

Functional neuroimaging has provided much of the physiological database about psychiatric disorders, particularly schizophrenia (Berman and Weinberger 1991; Andreasen et al. 1992; Liddle 1995). However, nuclear medicine techniques, such as single photon emission computed tomography (SPECT) and positron emission tomography (PET), tend to rely on group averaging and miss subtle interindividual differences, which may be 
as critical to understanding psychiatric disorders as are the group data. As a nonradioactive methodology, functional magnetic resonance imaging (fMRI) offers several advantages, including improved spatial and temporal resolution, virtually unlimited study repetitions, straightforward registration of functional and anatomic scans, and use on widely available MRI scanners. For these reasons, particularly repeatability, fMRI is an ideal individual mapping technique. As such, it may offer unique insights into several important questions, including the particular relationship between individuals and diagnostic groups, the effects of medication or performance differences on activation tasks, the distinction between state and trait findings, and the reliability of findings over time.

The fMRI literature has exploded over the past few years (Levin et al. 1995; Weinberger et al. 1996). From simple motor and sensory tasks to complex cognitive paradigms, our group (Duyn et al. 1994; Van Gelderen et al. 1995; Mattay et al. 1996; Ramsey et al. 1996a, b) and others (Belliveau et al. 1991; Bandettini et al. 1992; Kwong et al. 1992; Ogawa et al. 1992; Cao et al. 1993; McCarthy et al. 1993, 1994; Binder and Rao 1994; Cohen et al. 1994; Cohen and Brookheimer 1994; D'Esposito et al. 1995; Kwong 1995) have demonstrated the use of fMRI to detect regionally specific signal changes thought to indirectly represent neuronal activity. Yurgelun-Todd et al. (1996) recently demonstrated decreased left prefrontal cortical activation in patients with schizophrenia compared to healthy subjects during a verbal fluency task. Also utilizing fMRI, Breiter et al. (1996) reported activation of several limbic and paralimbic regions during symptom provocation in patients with obsessive-compulsive disorder.

The unique potential of fMRI is counterbalanced by a unique set of challenges in data acquisition, analysis, and interpretation. In general, any given signal change (or "activation") detected during a fMRI study is not necessarily related to neuronal activity; it may instead result from various, often subtle, artifacts. Some artifacts are inherent to the technique (e.g., "inflow," machine noise), whereas others will vary across individuals (e.g., brain motion, head movemert). Given the low physiological signal-to-noise ratio (SNR) of fMRI on conventional 1.5 Tesla $(1.5 \mathrm{~T})$ machines, it is typically by virtue of multiple stable repetitions of reference ("off") and activation ("on") states that signal will stand out against the background over time. Anything impinging on the stability of the response over time (voxel stability), such as motion, physiological variability, or machine artifact, will render data suspect in an unpredictable manner (i.e., either overactivation or underactivation) (Bullmore et al. 1996a; Eddy et al. 199í; Lee et al. 1996; Maas et al. 1997). Thus, the particular problem in applying this technique to neuropsychiatric illness will be recognizing and avoiding the artifactual "activation" (both increases and decreases) that will inevitably be found (Callicott and Weinberger, in press).

The goal of this study is twofold: (1) we describe a study of a novel working memory task in patients with schizophrenia; and (2) we illustrate a conservative approach to fMRI data that should be applicable to other fMRI studies. Numerous prior studies (for a review, Weinberger and Berman 1996) have addressed the possibility of prefrontal cortical dysfunction in patients with schizophrenia first suggested by the work of Ingvar and Franzen (Ingvar and Franzen 1974) and often broadly categorized under the term "hypofrontality." Whereas studies of resting blood flow or metabolism have been inconclusive, studies utilizing cognitive activation paradigms have tended to show that patients with schizophrenia underactivate prefrontal cortex in response to cognitive challenges (Weinberger and Berman 1996). Furthermore, this failure has been most robustly demonstrated in tasks, like the Wisconsin Card Sorting Task, thought to rely on working memory-the ability to hold information briefly "on-line" for manipulation or later storage (Baddeley 1996; Goldman-Rakic 1996). In the present study, we have utilized a novel version of the " $\mathrm{n}$ " back working memory task (Gevins et al. 1987). This task has been previously shown to activate prefrontal cortex in healthy controls (Cohen et al. 1994, 1997) and may be a more pure assay of working memory. We hypothesized that patients with schizophrenia would underactivate prefrontal regions during this working memory challenge when compared to healthy controls.

As part of our exploration of this hypothesis, we highlight a systematic and critical appraisal of our fMRI methods. This approach assumes the presence of artifact and aggressively seeks to identify and minimize its effects. Two key issues are emphasized. First, how do we assure ourselves that we have gathered valid physiological activation data? Second, how do we know differences between individuals and groups (e.g., hypofrontality in patients with schizophrenia) are not statistical artifacts? To address the first question, we incorporate a predicted motor response into the cognitive task. Thus, activation of sensorimotor cortex-a region that is probably functionally intact in psychiatric disorders (Bogerts 1993; Buckley et al. 1997; Mattay et al. 1997)-is expected and is used as a reference or control region ("internal standard") both within and across groups. To address the second question, we controlled across subjects and across groups for differences in signal variance or voxel stability. This provides a match for arbitrary group differences (regardless of their source) and ensures that variance characteristics will not systematically bias activation differences across groups. Whereas our approach is neither exhaustive nor meant as a "standard" for fMRI in psychiatric research, we demonstrate that failure to systematically address these general methodological issues jeopardizes the already com- 
plex process of evaluating data possibly related to the neurophysiology of mental illness.

\section{METHOD}

\section{Subjects}

We studied 10 male patients with schizophrenia (PT) $(10 / 10$ right-handed (Oldfield 1971), mean \pm SD age $=$ $34.1 \pm 6.2)$ and 10 male normal controls (NC) $(8 / 10$ right-handed, $33.3 \pm 7.6$ ) (see Table 1). Patients were recruited from the inpatient wards of the National Institute of Mental Health (NIMH) Neuropsychiatric Research Hospital at St. Elizabeths, Washington, DC. The Investigational Review Board of the NIMH approved the study protocol. All patients met Diagnostic and Statistical Manual IV (DSM-IV) criteria for schizophrenia and were screened to exclude concomitant neurological illness and active substance abuse. Normal volunteers were recruited from the National Institutes of Health (NIH) staff and from the community and were paid for their participation. After complete description of the study, written consent was obtained.

\section{Protocol}

The working memory paradigm consisted of a novel version of the "two back" working memory task com- bined with a "no back" control task. Our task differed from other versions (Cohen et al. 1994) in that a motor response was required for every stimulus. This motor response was designed to generate activation in contralateral sensorimotor cortex-a reference region. Stimuli were presented at a rate of $0.6 \mathrm{~Hz}$ (every $1.8 \mathrm{~s}$ ) on a rear projection screen placed at the subject's feet. A mirror attached to the head coil allowed subjects to see the screen. The stimuli consisted of number (one to four) shown in random sequences and displayed at the points of a diamond-shaped box (Figure 1). "No back" and "two back" conditions were alternated with "rest" periods during which subjects were asked to relax with eyes open.

The two back task required subjects to continually update their mental set while responding to previously seen stimuli. This kind of delayed-response task has been widely held to require working memory and has been demonstrated to activate the prefrontal cortex of human and nonhuman primates (Goldman-Rakic 1991; Cohen et al. 1994).

Working memory data were collected in "runs." Nine of these runs, with a brief pause between, were gathered over the course of an entire experiment that lasted $40 \mathrm{~min}$. Each run consisted of multiple task "epochs." Each epoch lasted $30 \mathrm{~s}$ and consisted of either the rest, no back, or two back conditions. Each condition was repeated twice during one run. Thus, each task condition was repeated 18 times over the entire study. The activation conditions were presented in a counterbalanced fashion to avoid order effects.

Table 1. Patient Characteristics

\begin{tabular}{|c|c|c|c|c|c|c|c|}
\hline $\begin{array}{l}\text { Patients with } \\
\text { Schizophrenia } \\
\text { (PT) }\end{array}$ & $\begin{array}{c}\text { Age/ } \\
\text { Handedness }\end{array}$ & $\begin{array}{c}\text { Diagnostic } \\
\text { Subtype }^{a}\end{array}$ & $\begin{array}{c}\text { Medications } \\
(\mathrm{mg} / \mathrm{d})\end{array}$ & $\begin{array}{c}\text { Duration }^{c} \\
\text { (years) }\end{array}$ & $\begin{array}{l}\text { PANSS }+^{d} \\
(\max =49)\end{array}$ & $\begin{array}{l}\text { PANSS }-d \\
(\max =49)\end{array}$ & $\begin{array}{c}\text { AIMS }^{d} \\
(\mathbf{m a x}=28)\end{array}$ \\
\hline 1 & $22 / R$ & PAR & $\begin{array}{l}\text { clozapine } 600 \\
\text { sertraline } 50\end{array}$ & 4 & 14 & 25 & 1 \\
\hline $2^{*}$ & $37 / \mathrm{R}$ & UND & $\begin{array}{l}\text { haloperidol } 8 \\
\text { benztropine } 2\end{array}$ & 17 & 9 & 33 & 2 \\
\hline $3^{*}$ & $27 / \mathrm{R}$ & PAR & $\begin{array}{l}\text { risperidone } 3 \\
\text { lithium } 900\end{array}$ & 8 & 7 & 11 & 0 \\
\hline 4 & $36 / \mathrm{R}$ & UND & risperidone 8 & 21 & 7 & 34 & 1 \\
\hline $5^{*}$ & $41 / \mathrm{R}$ & PAR & $\begin{array}{l}\text { haloperidol } 18 \\
\text { benztropine } 2\end{array}$ & 20 & 20 & 27 & 0 \\
\hline $6^{*}$ & $41 / \mathrm{R}$ & PAR & risperidone 6 & 17 & 13 & 14 & 0 \\
\hline 7 & $34 / \mathrm{R}$ & PAR & $\begin{array}{l}\text { clozapine } 550 \\
\text { valproate } 1000\end{array}$ & 17 & 15 & 18 & 1 \\
\hline $8^{*}$ & $29 / \mathrm{R}$ & PAR & NEVER & 6 & 21 & 9 & 0 \\
\hline 9 & $36 / R$ & PAR & OFF & 16 & 17 & 32 & 3 \\
\hline $10^{*}$ & $38 / \mathrm{R}$ & PAR & NEVER & 15 & 23 & 26 & 0 \\
\hline Mean $\pm(S D)$ & $34 \pm(6)$ & & & $14.1 \pm(6)$ & $14 \pm(5)$ & $23 \pm(9)$ & $0.8 \pm(1)$ \\
\hline
\end{tabular}

"Schizophrenia subtypes (DSM-IV) are abbreviated PAR for paranoid and UND for undifferentiated types.

"Patients \#8-10 were medication-free at the time of scan, whereas the remaining patients had been at the reported dose for greater than 6 weeks. PT \#9 had beer taken off all medications for 3 weeks, whereas PTs \#8 and 10 had never received neuroleptics.

'Duration of illness is defined as the time elapsed since onset of psychotic symptoms.

"Patients were rated at the time of scan by two psychiatrists using the positive and negative symptom scale (Kay 1991) (PANSS + and PANSS subscales) and the abnormal and involuntary movement scale (AIMS) modified (Wyatt 1993). Ratings were highly correlated between raters, with intra-class correlations of $0.93,0.76$, and 0.89 , respectively.

* Refers to patients retained for the final analysis. 


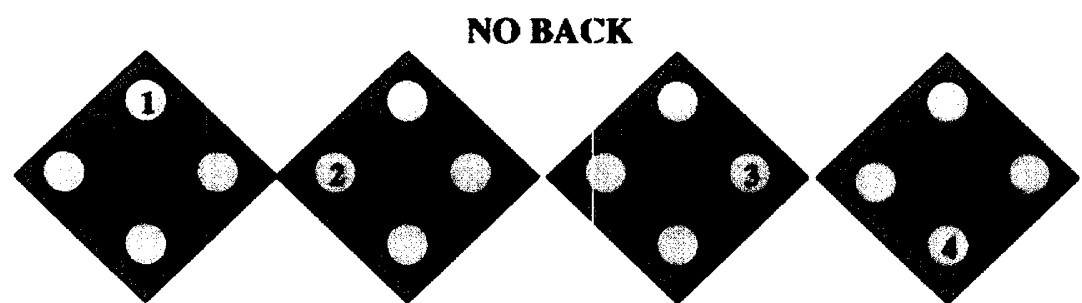

RESPONSE: 1

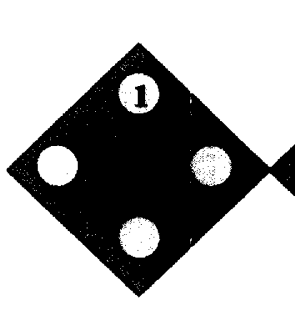

RESPONSE: -
RESPONSE: 2
RESPONSE: 3

TIME $\longrightarrow$

Prior to being placed in the scanner, each participant reviewed the rules and practiced each condition. To limit movement, subjects were placed on a rolling platform with their heads suspended in a hammock-like head-
Figure 1. Working memory activation task rules: The numbers 1-4 were displayed randomly. Each number had its own unique and fixed position on the four corners of the diamond. Responses were recorded using a button box with buttons arrayed in the same configuration. Subjects responded by pressing with their right thumb one of these four buttons. These responses were recorded by computer. During the no-back condition, the subject pressed the button corresponding to the number seen on the screen. During the two-back condition, the subject was required to respond to the number seen two stimuli previously (i.e., "go back two numbers in the sequence for your response"). holder (Ramsey et al. 1996a, b). To acclimatize subjects to the scanner, they were given further practice inside the scanner with the pulse sequence running in the background.

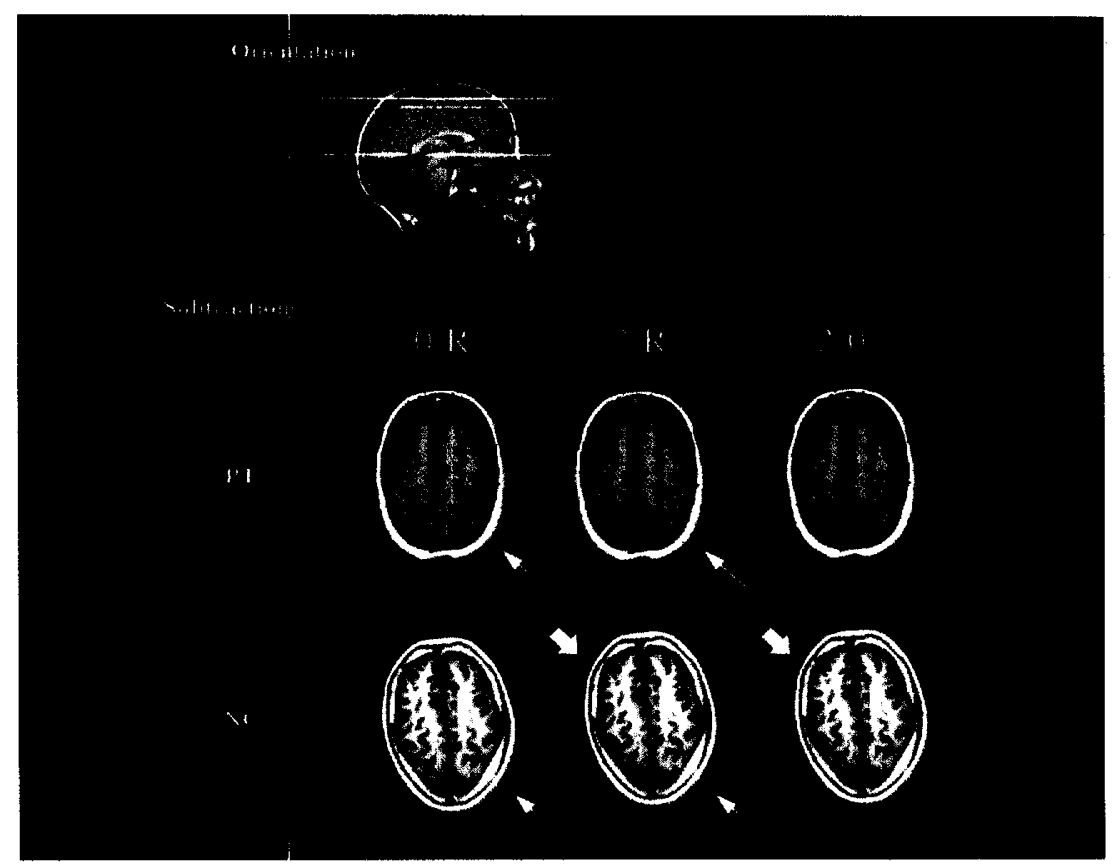

Figure 2. Individual $\mathrm{fMRI}$ activation maps for a patient with schizophrenia (PT) and a matched control (NC) during a "two-back" (2B) working memory (WM) task compared with an eyes-open "rest" (R) and "no-back" control task (0B). fMRI data (boundaries indicated by jagged white line) were superimposed on oblique Inversion Recovery images. Upper figure denotes orientation of 3-D fMRI slab (thick white line) with respect to the anterior commissure-posterior commissure (AC-PC) line (red line). Yellow line indicates approximate location of slices shown below. Lower figure contains a representative slice from each subject encompassing the sensorimotor reference region (SM) and dorsolateral prefrontal cortex (DLPFC). Three task substraction comparisons are shown. highlighting SM activation ( $0-\mathrm{R}$ and $2-\mathrm{R})$ and WM activation (2-0). Red voxels highlight positive activated voxels exceeding critical threshold. Although both subjects have SM activation (small arrows), only the NC activated DLPFC (large arrows). 


\section{Data Acquisition}

We used an fMRI technique called principles of echo shifting with a train of observations (PRESTO) (Liu et al. 1993; Van Gelderen et al. 1995; Ramsey et al. 1996a, b) - a hybrid of echo-planar imaging (EPI) and fast low angle shot (FLASH) imaging. At this tirne, most BOLD-based fMRI techniques represent modifications of EPI or FLASH-each with its own particular strengths and weaknesses in spatial and temporal resolution. SNR, and hardware requirements. Particular advantages of PRESTO include the acquisition of a true isotropic threedimensional (3-D) volume, allowing for both straightforward registration, transposition of functional data with anatomic images obtained in any plane, and reduced susceptibility to so-called "inflow effects" (Duyn et al. 1994; Van Gelderen et al. 1995). Also, PRIESTO can be used on a standard $1.5 \mathrm{~T}$ scanner without additional hardware. The details of the PRESTO sequence and imaging protocol are presented elsewhere (Van Gelderen et al. 1995; Ramsey et al. 1996a, b). A particular disadvantage of 3-D PRESTO is that the time needed to acquire a full $3-D$ volume $(6 \mathrm{~s})$ allows more time for subjects to move ("within scan movement") than single-shot 2-D methods like EPI that rapidly acquire single slices (e.g., TE $=: 40-60 \mathrm{~ms}$ ). However, because multiple single slices are required to cover the same volume of brain as PRESTO, subject movement in the interval between these slices ("between scan" motion) is potentially more problematic (Weinberger et al. 1996). A detailed comparison of these techniques is beyond the scope of this article; however, these particular strengths or weaknesses must be taken into account in the design and analysis of $\mathrm{fMRI}$ experiments.

\section{Image Registration}

Misregistration secondary to between-scan movements can be particularly detrimental to functional imaging studies. Small movements can generate large apparent signal differences, especially in voxels located near highcontrast edges. During each 30-s epoch, five 3-D fMRI volumes (each volume comprising approximately 11,000 , $3.75-\mathrm{mm}$ isotropic voxels) were acquired. The first volume of each epoch was discarded to allow for hemodynamic delay in the BOLD effect (Cgawa et al. 1990). The remaining volumes from all epochs were registered to the last volume in the session using a least-squares differences algorithm and cubic spline interpolation (Unser et al. 1993). A cine loop composed of a sagittal slice from each of the registered volumes was used to visually confirm proper alignment.

\section{Creation of Individual Activation Maps by the Subtraction Method}

For each individual subject, datasets were created by various subtraction comparisons (Binder et al. 1995) (see below) that were analyzed statistically using the $\mathrm{z}_{\mathrm{t}}$-map method described previously (Van Gelderen et al. 1995; Ramsey et al. 1996b). After registration, the four volumes (i.e., the 4-D array of voxels) from each epoch were averaged. This "averaged volume" represented data during one repetition of a condition-either rest, no back, or two back. Since each working memory condition was repeated twice during a run and 18 times during the nine runs, there were 18 "averaged" fMRI volumes for each task condition. The 18 "averaged volumes" from each condition were then used to perform task substraction comparisons.

To measure activation related to sensorimotor function, rest "averaged volumes" were subtracted on a voxelby-voxel basis from the no-back and two-back "averaged volumes" (e.g., no back minus rest [0B - R] and two back minus rest $[2 \mathrm{~B}-\mathrm{R}])$. To examine signal changes specific to working memory, the no-back volumes were subtracted from the two-back volumes $(2 \mathrm{~B}-0 \mathrm{~B})$. The resultant signal change differences for each voxel comprised a "difference volume."

For each voxel in these "difference volumes," a mean signal change and standard deviation were calculated. A $\chi^{2}$ test using the formula [(Voxel variance) $\times \sqrt{ }$ (number of task epochs)]/(pooled variance)] identified those voxels exhibiting a large variance ( $\chi^{2}$ threshold corresponding to $p=.01$ corrected for the total number of voxels) that are likely to include artifacts, e.g., from large vessels or within-scan movement (Van Gelderen et al. 1995). We have previously shown that these voxels represent less than $1 \%$ of the total number of PRESTO voxels (Van Gelderen et al. 1995; Ramsey et al. 1996b). A pooled standard deviations (PSD) was created from the remaining voxels. A separate PSD was calculated for the subgroup of high variance voxels to establish an appropriately stringent threshold for these voxels. The PSD was used to calculate a $z_{\mathrm{t}}$-value for all voxels by multiplying each voxel's mean signal change by [ $V$ (number of difference volumes)/PSD]. Finally, activated voxels were identified as those crossing a critical statistical threshold. The critical $z_{t}$ threshold was defined separately for each subject by applying a Bonferroni correction for the total number of voxels in the entire 3-D volume (approximately 11,000 voxels). This resulted in $z_{t}$ thresholds between $4.4-4.5$ $\left(p<4.4-4.5 \times 10^{-6}\right.$, omnibus $p<.05$ (one-sided)). Voxels crossing this threshold were highlighted and manually overlaid on the oblique inversion recovery (IR) images for localization $\left(z_{\text {crit }}\right.$ map). Only voxels with a positive signal change were analyzed and are reported herein simply as the number of activated voxels.

\section{Group Comparisons}

Using the $z_{\text {crit }}$ maps, activated voxels were assigned to anatomic regions based on their location on the oblique 
IR images (Watson 1991; Talairach and Tournoux 1993) (Figure 2). The sensorimotor control region (SM) was defined liberally-encompassing, on all relevant slices, the areas surrounding the central sulcus and including precentral gyrus (BA 4), lateral aspects of premotor area (BA 6), and postcentral gyrus (BA 1-3.5). DLPFC was determined by examining the middle and inferior frontal gyri superior and rostral to the genu of the corpus callosum on all relevant slices (putative BA 10,46). Whereas the oblique slab orientation maximized frontal coverage, only the most anterior and superior aspects of the parietal cortex (PAR) were included (putative BA 7,40).

Group differences were compared by Mann-Whitney $\mathrm{U}$ (two-tailed $p$ ) and Spearman's rank-order correlation tests. The group data are hereafter reported as medians. Data for DLPFC and PAR are presented as the sum of right and left cortices. Data for SM are taken from the left cortex (contralateral to right thumb movement). Group differences in voxel stability (see below), in contrast, were based on normally distributed difference values within each individual and were therefore compared using Student's $t$-test for independent samples (two-tailed $p$ ).

\section{RESULTS}

The individual maps showed that most patients with schizophrenia $(9 / 10)$ failed to have any "activated" voxels in DLPFC during the "two-back" task (Figure 2). Accordingly, in the $2 \mathrm{~B}-\mathrm{OB}$ comparison, normal controls showed significantly greater "activation" in DLPFC with a median number of significant voxels of 5.0 compared with 0 for the patients $\left(\mathrm{U}=17.5, \mathrm{n}_{2}=10, p=.01\right)$. At the same time, however, median activation in the contralateral SM control region also tended to be different between the groups in both the $0 \mathrm{~B}-\mathrm{R}$. comparison ( 8.5 versus $4, \mathrm{U}=30.0, \mathrm{n}_{2}=10, p=.1$ ) and in the $2 \mathrm{~B}-\mathrm{R}$ comparison (7.0 vs. $2.0, \mathrm{U}=25.5, \mathrm{n}_{2}=10, p=.06$ ), even though the groups had almost the same number of motor responses. Several patients and controls failed to show any activation in the left $\mathrm{SM}$ reference region during the $0 \mathrm{~B}-\mathrm{R}$ (PTs \#1,4,9 and $\mathrm{NC} \# 8$ ) and $2 \mathrm{~B}-\mathrm{R}$ comparisons (PTs \#1,8,9; NC\#10). In other words, to the extent that activation of SM should have been found, our "internal standard" suggested that there was a problem with the validity of the "assay."

\section{Matching for Voxel Stability}

Since differences in the number of activated voxels could be explained by either group differences in mean signal change or in mean variance (or both), we next examined differences in a measure of voxel stability (PSD). Perhaps not surprisingly, the patients with schizophrenia showed significantly greater voxel instability. In the $O B-R$ and $2 B-R$ comparisons, patients had signifi- cantly greater PSD than controls $(2.1 \pm 0.7$ vs. $1.4 \pm 0.4$, $t=2.2, d f=18, p=.04)$. Similarly, in $2 \mathrm{~B}-0 \mathrm{~B}$, patients had greater PSD $(1.9 \pm 0.7$ vs. $1.4 \pm 0.3, t=2.4, d f=18$, $p=.04)$. The five subjects who failed to activate SM had significantly higher mean PSD than did the other 15 subjects ( $2.4 \pm 0.8$ vs. $1.6 \pm 0.4, t=2.8, d f=18, p=.01)$. Thus, a relative lack of "activation" appeared to be related to this stability difference. To control for this artifact, subjects were then matched pairwise for PSD, resulting in rarefied samples of six each, that no longer differed in PSD in any task comparison (all $p>.6$ ).

As in the larger sample, all but one patient failed to activate $D L P F C$ in the $2 B-O B$ comparison and controls as a group had significantly greater activation ( 5.0 vs. $0, \mathrm{U}=$ $1.0, \mathrm{n}_{2}=6, p=.006$ ) (see Table 2). Now, however, all subjects showed SM activation in the $0 \mathrm{~B}-\mathrm{R}$ comparisons and all but one (PT \#8) showed significant SM activation in the $2 B-R$ comparison. No significant group differences in $\mathrm{SM}$ were found in either comparison. Thus, controlling for variance restored the internal activation standard.

Most subjects also activated parietal cortex during the motor control and the working memory tasks. Though patients and controls did not differ significantly in the amount of activation, the PAR data suggested contrasting activation patterns. In the $O B-R$ and $2 B-R$ comparisons, all but two subjects (PT \#3 in $0 B-R$ and PT $\# 6$ in $0 B-R$ and $2 B-R$ ) showed significant activation in PAR. Similarly, all but three subjects NC \#3, PTs \#8 and $\# 10$ ) activated PAR in the $2 B-O B$ comparison. However, whereas controls had more PAR activated voxels than did patients in $0 B-R(13.5$ vs. 2.5$)$ and $2 B-R$ (17.5 vs. 4.5$)$, patients had more PAR activation in the $2 \mathrm{~B}-0 \mathrm{~B}$ working memory comparison ( 7.5 vs. 1.0 ).

The two groups did not differ in performance on the no back control task. While patient performance was poorer during two back (controls correct $11.5 \pm 2.5$ vs. patients $5.1 \pm 3.8, \mathrm{~F}=11.8, d f=1, p=.006$ ), there was no significant correlation between performance and the number of activated voxels in any region. The availability of individual activation data made it possible to explore further the relationship between activation and performance. PT \#2, the only patient who activated DLPFC in the $2 \mathrm{~B}-\mathrm{OB}$ comparison, had a mean performance of $4.1 \pm 1.6-$ well below the range for controls (7.6-13.3) and fourth of sixth in patient performance. In contrast, PT \#8 had the highest mean performance among the patients at $12.5 \pm 1.5$-well within the normal range-yet failed to activate DLPFC. In addition, NC \#2 had the lowest mean performance among controls at $7.6 \pm 2.0$, yet still activated DLPFC in the $2 \mathrm{~B}-0 \mathrm{~B}$ comparison.

\section{DISCUSSION}

"fMRI" refers to an array of methodologies, encompassing different pulse sequences, slice characteristics (e.g., 
Table 2. Number of Positive "Activated" Voxels ${ }^{a}$ from Motor Control (No Back - Rest, 2 Back - Rest) and Working Memory (2 Back - No Back) Task Subtraction Comparisons: Matched Sample

\begin{tabular}{|c|c|c|c|c|c|c|c|c|c|c|c|c|}
\hline \multirow[b]{4}{*}{ Subject } & \multicolumn{8}{|c|}{ Motor Control Comparisons } & \multirow{2}{*}{\multicolumn{4}{|c|}{$\frac{\text { Working Memory Comparisons }}{2 \text { Back - No Back }}$}} \\
\hline & \multicolumn{4}{|c|}{ No Back - Rest } & \multicolumn{4}{|c|}{2 Back - Rest } & & & & \\
\hline & \multicolumn{2}{|c|}{$\mathbf{S M}^{b}$} & \multicolumn{2}{|c|}{ Par } & \multicolumn{2}{|c|}{ SM } & \multicolumn{2}{|c|}{ Par } & \multicolumn{2}{|c|}{ DLPFC } & \multicolumn{2}{|c|}{ Par } \\
\hline & $\mathbf{R}^{d}$ & $\mathbf{L}$ & $\mathbf{R}$ & $\mathbf{L}$ & $\mathbf{R}$ & $\mathbf{L}$ & $\mathbf{R}$ & $\mathbf{L}$ & $\mathbf{R}$ & $\mathrm{L}$ & $\mathbf{R}$ & $\mathbf{L}$ \\
\hline $\mathrm{NC} 1$ & 3 & 12 & - & 3 & 17 & 23 & 1 & 3 & 5 & - & 2 & - \\
\hline NC2 & 4 & 5 & 4 & 4 & 3 & 1 & 5 & 8 & 10 & 8 & 39 & 4 \\
\hline NC3 & - & 10 & - & 3 & 1 & 7 & 7 & 5 & 6 & 1 & - & - \\
\hline $\mathrm{NC4}$ & 1 & 7 & 10 & 22 & 1 & 6 & 12 & 26 & 1 & - & 1 & - \\
\hline NC5 & 22 & 20 & 13 & 6 & 15 & 19 & 15 & 7 & 5 & 5 & 1 & - \\
\hline NC6 & 12 & 4 & 19 & 3 & 4 & 7 & 47 & 8 & - & 5 & - & 1 \\
\hline PT2 & 8 & 18 & '73 & 22 & 13 & 16 & 80 & 23 & - & - & 51 & 10 \\
\hline РТ3 & 3 & 19 & - & - & 6 & 7 & 12 & 4 & 2 & - & 10 & 2 \\
\hline PT5 & 2 & 6 & 2 & - & 1 & 4 & 1 & - & - & - & 14 & - \\
\hline PT6 & - & 3 & - & - & 2 & 3 & - & - & - & - & 2 & 1 \\
\hline PT8 & - & 3 & 6 & 2 & 9 & - & 3 & 2 & - & - & - & - \\
\hline PT10 & - & 5 & - & 3 & - & 1 & 1 & 3 & - & - & - & - \\
\hline
\end{tabular}

${ }^{a}$ Mean number of voxels crossing conservative, Bonferroni-corrected $z_{t}$ threshold (see text).

${ }^{b} \mathrm{SM}=$ sensory + motor cortices; PAR $=$ parietal cortex; DLPFC $=$ dorsolateral prefrontal cortex

${ }^{c} \mathrm{NC}=$ normal controls; $\mathrm{PT}=$ patient with Schizophrenia; PT\# refers to Table 1.

${ }^{d} \mathrm{R}$ and $\mathrm{L}$ refer to right and left hemisperes.

3-D vs. 2-D, single vs. multislice, orientation, voxel dimensions), magnet strength, and data processing algorithms. Amidst this diversity, there are confounds common to all fMRI methods, some of which are likely exaggerated in psychiatric patients. In general, all fMRI data are subject to artifacts that can generate spurious data both in terms of overactivation and underactivation. All fMRI techniques must overcorne low physiological SNR. At $1.5 \mathrm{~T}$, the signal changes measured as "activation" with BOLD fMRI range from 1\%-5\% (Bandettini et al. 1992; Cao et al. 1993; Ogawa et al. 1993). Therefore, the scans must be repeated many times to gain statistical power. Voxels that show signal increases in response to a task may cross some arbitrary statistical threshold and be called "activation." Anything interfering with this process, such as subject movement (i.e., changing the position of this voxel in imaging space) or machine variability, will affect the results. Given a certain level of unavoidable artifact, it is crucial to develop guidelines to minimize and control for these effects.

Based on our experience and a growing body of literature devoted to separating fMRI activation from artifact (Weinberger et al. 1996), we have adopted a conservative approach to evaluating fMRI data (Table 3 ). We will discuss the results of our working memory study in the context of this approach. The first stage of this process is prevention. In this report, we have not specifically addressed the assessment of machine artifact or physiological variability, but rather introduce these topics as they represent areas of active study likely to be important in future fMRI reports. A more problematic artifact in fMRI data is motion. Although it is clear that motion cannot explain all activation (Weisskoff 1995), movement has been shown to account for significant signal changes (Hajnal et al. 1994, 1995) and can be the source of subtle differences between populations-particularly with neuropsychiatric patients-even when utilizing a rigorous statistical threshold such as ours. In an attempt to prevent motion, we utilized a hammocklike head immobilization device that was well tolerated by patients and controls alike. Clearly, however, motion remained a factor in much, if not all, of these data. Other groups have experimented with bite blocks, foam pads, and inflatable head cushions with mixed results in terms of immobilization and subject comfort (Baudendistel et al. 1996; Lo et al. 1996). In our experience, it is difficult to find data unaffected by motion, even in cooperative healthy controls, thus necessitating further assessment of all data.

The second stage of evaluation (Table 3) involves various "quality control" measures that seek to minimize the effect of artifacts hidden in the data. The initial step in preparing fMRI data for analysis is the registration process, whereby data taken across the duration of an experiment are brought into a common image space by adjusting for movements between each acquisition. Subject movement can occur both within a given scan and between consecutive scans. Within scan movement is not correctable, thus pulse sequences acquiring functional images ("timepoints") over a longer time are already at a relative disadvantage, since more time is available for subject movement. It is also important to note 
Table 3. Structured Approach to Evaluation of fMRI Data

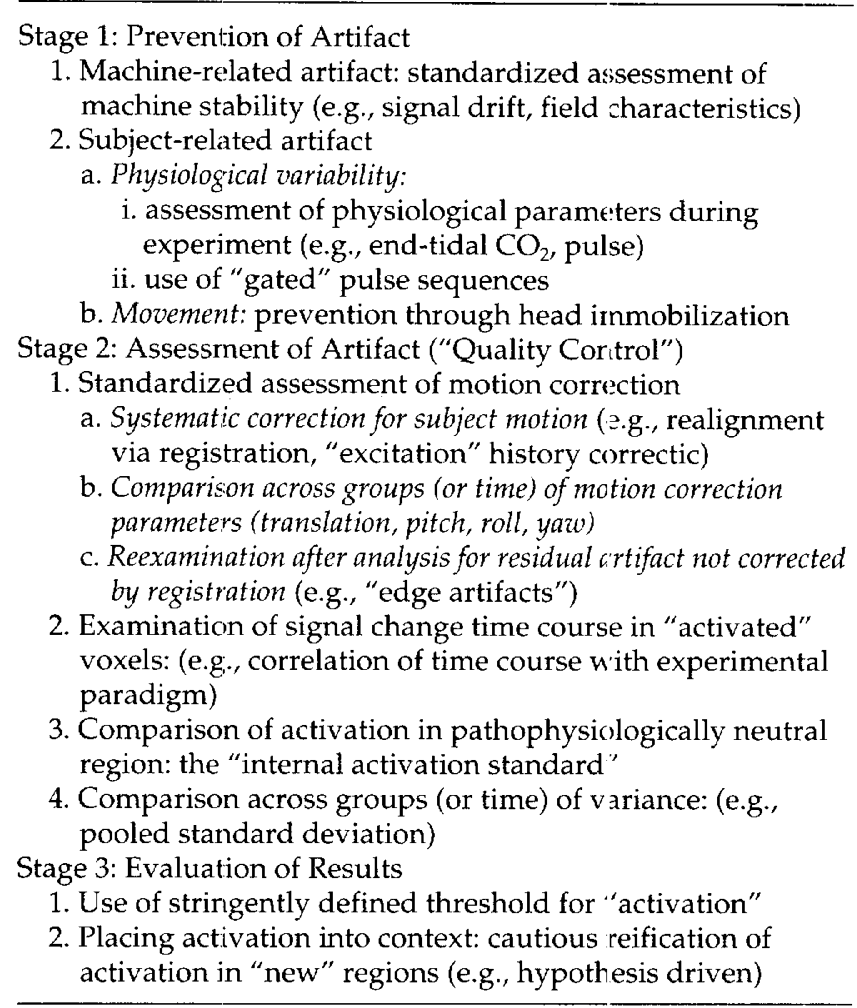

that although between-scan movement is correctable to a degree, these re-registration corrections are approximations that alter the data (Hajnal et al. 1994; Aine 1995). Furthermore, failure to register or align image data can be responsible for both increases and decreases in signal change. PRESTO, as a 3-D vclume, possesses certain advantages over single or multiple slice techniques in that it allows for straightforward correction of movements in all directions, whereas only movement within the plane of scan is correctable with single or multislice acquisitions that do not cover the whole brain.

Regardless of method, the registration process should begin with some systematic assessment of the presence of between-scan movement. It is becoming standard practice to report the amount of movement in 2-D imaging protocols such as EPI, most cornmonly derived from the amount of correction made by the registration algorithm (the "interpolation parameters") (Santha et al. 1997). Instead, we assume that any motion will be reflected in increased variance or voxel instability (i.e., higher PSD). In a more recent study using EPI in patients with schizophrenia (Callicott et al. 1997), we utilized both quantification of movement and voxel variance and have found the latter to be a good correlate of the former.

The final two aspects of quality control, the comparison of activation in a reference region and of voxel sta- bility (PSD), have been stressed in this report. A reference or control region is particularly useful because it provides an immediate qualitative gestalt regarding the final product-the functional maps. Group discrepancies in activation of this reference region should alert one to subtle or unforeseen sources of group dissimilarity (e.g., voxel stability characteristics, inherent physiological reactivity) that must be taken into account before activation differences in principal regions of interest are attributed to disease-specific neurophysiological abnormalities.

The final aspect of our approach to quality control is to use the pooled standard deviation to identify those subjects with excessive variance across scans and to match groups for statistical comparisons. In general, activation is a statistical representation of a numerator consisting of some changing parameter (here, signal intensity change) and a denominator consisting of a variance term. The increased spatial and temporal resolution of $\mathrm{fMRI}$ - usually touted as major advantages-can generate subtle, but profound differences in this variance term within individuals and especially between groups. Thus, matching for PSD (or variance) allows one to compare groups while correcting for statistical power within subjects. In order to make statements about between group differences, one must consider ways to match for statistical power, particularly with fMRI in which this power is hidden in the within-subject analysis. Using a variable like PSD gives readers insight into the quality of the individual data after the complete sequence of processing steps. On the other hand, one limitation of our matching technique is that the PSD essentially pools all sources of variance. It may ultimately be useful to differentiate the various sources of variance, such as motion or physiological variability (Bullmore et al. 1996; Friston et al. 1996). Even so, a statistical analysis across groups will have to ensure that the statistical parameters are comparable.

Though our PSD-matching technique resulted in the exclusion of a sizable portion of our original patient sample, we argue that this design detail is crucial to the interpretation of fMRI data. The loss of subject data is outweighed by avoiding a more serious error of attributing group differences arising from methodology to differences in underlying neurophysiology. Prior to the stability match, the patients had less activation in the SM cortex. Although this finding has been reported (Wenz et al. 1994; Schroder et al. 1995), our experience clearly demonstrates that failure to control for other sources of group difference (as reflected in PSD) generated unreliable results and may explain these earlier findings in the fMRI literature. Ultimately, the persistence of a group difference between NC and PT in DLPFC activation argues for its robustness.

The final stage of fMRI data analysis (see Table 3) involves testing for significance. Strictly speaking, the is- 
sue of statistical thresholds should be settled a priori as part of the experimental design. The fMRI literature, however, has witnessed a variety of idiosyncratic approaches to defining statistical thresholds, often based on post hoc assessment of the data (i.e., "how do you make the best map?"). It is important to note that thresholds for fMRI activation are discretionary and thus should be defined stringently. The $z_{t}$ analysis method uses a conservative Bonferroni correction for the total number of voxels imaged (approximately 11,000 ). Voxelby-voxel comparisons, for example 'with multiple $t$-tests, without correction are particularly sensitive to identifying false positive signal changes given such large numbers of comparisons (type I error).

Our results demonstrate that, in contrast to normal subjects, patients with schizophrenia fail to activate DLPFC during a conceptually simple working memory task that, unlike earlier tasks (e.g., the Wisconsin Card Sorting Task, the Tower of Hanoi, and others), requires no problem solving and may more closely approximate the "transient relevance" central to working memory (Goldman-Rakic 1991). As a validation of earlier functional neuroimaging findings in sichizophrenia, these results further imply that the neurophysiological deficit in schizophrenia is relatively regional, at least during a working memory task. The basic mechanism of this deficit is uncertain. Recent data suggest that patients are able to appropriately activate prefrontal cortex at lower levels of working memory load (i.e., one back) but may have reduced capacity compared with healthy controls to activate prefrontal cortex in response at higher loads (i.e., two back) (Callicott et al. 1997).

The two most common criticisms of cognitive activation studies in schizophrenia have been that poorer patient performance and/or medication effects underlie the phenomenon of "hypofrontality." Because a full discussion of these complex issues is beyond the scope of this study, the reader is referred elsewhere for more detailed discussion (Weinberger and Berman 1996). Although we cannot rule out in this study poor patient performance as a factor, our individual mapping data together with the accumulation of data from many sources (Weinberger and Berman 1996) argue against this simple explanation. The role of medication also cannot be ruled out in this experiment. However, the observation that patients had similar PSM activation and a trend toward overactivation of the parietal cortex would be difficult to explain if one hypothesizes a globally disruptive effect of antipsychotic medications. It is also important to note that the never-medicated patients in this study were similar to patients on medication, consistent with earlier studies. (Andreasen et al. 1992; Rubin et al. 1991; Catafau et al. 1994). Clearly, these issues warrant further study in larger patient samples.

Whereas our results echo earlier prefrontal physiology findings in schizophrenia, we now have the ability to assess individual data on cognition-linked brain activation in patient populations. The contrasting patterns of PAR activation and the complex relationship between activation to performance are examples of the unique results made possible by $\mathrm{fMRI}$-generated individual datasets. As we have emphasized, however, this ability rests squarely upon systematic efforts to control for the effects of artifact. As fMRI is increasingly applied to the study of neuropsychiatry, especially schizophrenia (Renshaw et al. 1994; Wenz et al. 1994; Schroder et al. 1995; Yurgelun-Todd et al. 1996), systematic approaches to minimizing artifacts inherent in the technique-as illustrated with our sensorimotor activation data-will make these results more reliable. In conclusion, we have demonstrated that a conservative approach to the evaluation of $\mathrm{fMRI}$ data is especially necessary in psychiatry's challenging patient populations.

\section{ACKNOWLEDGMENTS}

We gratefully acknowledge Attanagoda K.S. Santha, Ph.D., Jeffrey Houtari, B.A., and Michael S. Myslobodsky, M.D., Ph.D. for their assistance.

\section{REFERENCES}

Aine C (1995): A conceptual overview and critique of functional neuroimaging techniques in humans: I. MRI/ fMRI and PET. Critical Reviews in Neurobiology 9:229309

Andreasen NC, Rezai K, Alliger R, Swayze VW, Flaum M, Kirchner P, Cohen G, O'Leary DS (1992): Hypofrontality in neuroleptic-naive patients and in patients with chronic schizophrenia: Assessment with Xenon 133 single-photon emission computed tomography and the tower of London. Arch Gen Psychiatry 49:943-958

Baddeley A (1996): The fractionation of working memory. Proc Natl Acad Sci USA 93:13468-13472

Bandettini PA, Jesmanowicz A, Wong EC, Hyde JS (1993): Processing strategies for time-course data sets in functional MRI of the human brain. Magn Reson Med 30(2):161-173

Bandettini PA, Wong EC, Hinks RS, Tikofsky RS, Hyde JS (1992): Time course EPI of human brain function during task activation. Magn Reson Med 25(2):390-397

Baudendistel K, Schad L, Friedlinger M, Wenz F, Essig M, Lorenz W (1996): fMRI image registration using different head fixation techniques. New York, International Society for Magnetic Resonance in Medicine

Belliveau JW, Kennedy DN, McKinstry RC (1991): Functional mapping of the human visual cortex by magnetic resonance imaging. Science 254:716-719

Berman KF, Weinberger DR (1991): Functional localization in the brain in schizophrenia. In Tasman A and Goldfinger SM (eds), American Psychiatric Press Review of Psychiatry, vol 10. Washington, DC, APA Press, pp 24-59 
Binder JR, Rao SM (1994): Human brain mapping with functional magnetic resonance imaging. In Localization and neuroimaging in neuropsychology. New York, Academic Press, Inc., pp 185-212

Binder JR, Rao SM, Hammeke TA, Frost JA, Bandettini PA, Jesmanowicz A, Hyde JS (1995): Lateralized human brain language systems demonstrated by task subtraction functional magnetic resonance imaging. Arch Neurol 52:593-601

Bogerts BH (1993): The neuropathology of schizophrenia. Schizophr Bull 19:68-75

Breiter HC, Rauch SL, Kwong KK, Baker IR, Weisskoff RM, Kennedy DN, Kendrick AD, Davis TL, Jiang A, Cohen MS, Stern CE, Belliveau JW, Baer L, C'Sullivan RL, Savage CR, Jenike MA, Rosen BR (1996): Functional magnetic resonance imaging of symptom provocation in obsessive-compulsive disorder. Arch Gen Psychiatry 53:595-606

Buckley PF, Friedman L, Wu D, Lai S, Meltzer HY, Haacke EM, Miller D, Lewis JS (1997): Functional magnetic resonance imaging in schizophrenia: Initial methodology and evaluation of the motor cortex. Psychiatr Res Neuroimaging 74:13-23

Bullmore E, Brammer M, Williams SC, Rabe R (1996a): Statistical methods of estimation and inference for functional MR image analysis. Magn Reson Med 35:261-277

Bullmore ET, Rabe-Hesketh S, Morris RG, Williams SCR, Gregory L, Gray JA, Brammer MJ (1996b): Functional magnetic resonance image analysis of a large-scale neurocognitive network. Neuroimage 4:16-33

Callicott JH, Bertolino A, Mattay VS, Santha AK, Finn K, Coppola RC, Goldberg TE, Frank JA, Weinberger DR (1997): fMRI of a parametric working memory task in schizophrenia. Biol Psychiatry 41:11S-12S

Callicott JH, Weinberger DR (in press). Functional brain imaging: Future prospects for clinical practice. In Weissman S, Sabshin M, Eist H (eds), 21st Century Psychiatry: The Foundations. Washington, DC, American Psychiatric Press

Cao Y, Towle vL, Levine DN, Balter JM (1993): Functional mapping of human brain cortical activation by conventional MRI at 1.5 T. J Magn Reson Med 3:869-875

Catafau AM, Faradella E, Lomena FJ, Bernardo M, Pavia J, Ros D, Setoain J, Gonzalez-Monclus E (1994): Prefrontal and temporal blood flow in schizophrenia: Resting and activation technetium-99m-HMPAO SPECT patterns in young neuroleptic-naive patients with acute disease. J Nucl Med 35:935-941

Cohen JD, Forman SD, Braver TS, Casey BJ, ServanSchreiber D, Noll DC (1994): Activation of the prefrontal cortex in a nonspatial working memory task with functional MRI. Human Brain Mapping 1:293-304

Cohen JD, Perstein WM, Braver TS, Nystrom LE, Noll DC, Jonides J, Smith EE (1997): Temporal clynamics of brain activation during a working memory task. Nature 386:604-608

D'Esposito M, Detre JA, Alsop DC, Shin RK, Atlas S, Grossman $M$ (1995): The neural basis of the central executive system of working memory. Nature 378:279-281

Duyn JH, Mattay VS, Sexton RH, Sobering GS, Barrios FA, Liu G, Frank JA, Weinberger DR, Moonen CT (1994): 3-dimensional functional imaging of human brain using echo-shifted FLASH MRI [published erratum appears in Magn Reson Med 1994 Oct; 32(4):545]. Magn Reson Med 32(1):150-155

Eddy WF, Fitzgerald M, Noll DC (1996): Improved image registration by using Fourier interpolation. Magn Reson Med 36(6):923-931

Friston KJ, Williams S, Howard R, Frackowiak RSJ, Turner R (1996): Movement-related effects of fMRI time-series. Magn Reson Med 35:346-355

Gevins AS, Morgan NH, Bressler SL, Cutillo BA, White RM, Illes J, Greer DS, Doyle JC, Zeitlin GM (1987): Human neuroelectric patterns predict performance accuracy. Science 235(4788):580-585

Goldman-Rakic PS (1990): Cellular and circuit basis of working memory in prefrontal cortex of nonhuman primates. In Uylings II, Van Eden G, DeBruin J, Corner $M$ and Feenstra M (eds), Progress in Brain Research, vol 85, New York, Elsevier, pp 325-336

Goldman-Rakic PS (1996): Regional and cellular fractionation of working memory. Proc Natl Acad Sci USA 93:13473-13480

Goldman-Rakic PS, Friedman HR (1991): Frontal lobe function and dysfunction. Levin HS and Eisenberg HM (eds), New York, Oxford University Press, pp 72-91

Hajnal JV, Bydder GM, Young IR (1995): fMRI: does correlation imply activation? NMR Biomed 8:97-100

Hajnal JV, Myers R, Oatridge A, Schwieso JE, Young IR, Bydder GM (1994): Artifacts due to stimulus correlated motion in functional imaging of the brain. Mag Reson Med 31:283-291

Ingvar D, Franzen G (1974): Distribution of cerebral activity in chronic schizophrenia. Lancet 2:1484-1486

Kay SR (1991): Positive and negative symptoms in schizophrenia: assessment and research, New York, Bruner Mazel

Kwong KK (1995): Functional magnetic resonance imaging with echo planar imaging. Magnetic Resonance Quarterly $11(1): 1-20$

Kwong KK, Belliveau JW, Chesler DA, Goldberg IE, Weisskoff RM, Poncelet BP, Kennedy DN, Hoppel BE, Cohen MS, Turner R, et al. (1992): Dynamic magnetic resonance imaging of human brain activity during primary sensory stimulation. Proc Natl Acad Sci USA 89(12): 5675-5679

Lee CC, Jack CR, Grimm RC, Rossman PJ, Felmlee JP, Ehman RL, Riederer SJ (1996): Real-time adaptive motion correction in functional MRI. Magn Reson Med 36(3): $436-444$

Levin J, Ross M, Renshaw P (1995): Clinical applications of functional MRI in neuropsychiatry. J Neuropsychiatry 7(4):511-522

Liddle P (1995): Brain imaging. In Hirsch S, Weinberger D (eds), Schizophrenia. London, Blackwood Press, pp 425-439

Liu G, Sobering G, Duyn J, Moonen CT (1993): A functional MRI technique combining principles of echo-shifting with a train of observations (PRESTO). Magn Reson Med 30(6):764-768

Lo K, Kwok E, Yamada K, Totterman S (1996). A novel head immobilization device for functional MRI. International Society for Magnetic Resonance in Medicine, New York 
Maas LC, Frederick BD, Renshaw I'F (1997): Decoupled automated rotational and translational registration for functional MRI time series data: The DART registration algorithm. Magn Reson Med 37(1):131-139

Mattay VS, Callicott JH, Bertolino AB, Santha AKS, Tallent KA, Frank JA, Weinberger DR. Anomalous lateralization of motor cortex function in patients with schizophrenia. Neuroreport [in press]

Mattay VS, Frank JA, Santha AKS, Pekar JJ, Duyn JH, McLaughlin AC, Weinberger DR. (1996): Whole brain functional mapping with isotropic MR imaging. Radiology 201:399-404

Mattay VS, Callicott JH, Bertolino A, Santha A, Tallent K, Frank J, Weinberger DR (1997): Sensorimotor cortex lateralization is anomalous in patients with schizophrenia: A whole brain fMRI study. Neuroimage 5:S23

McCarthy G, Blamire AM, Puce A, Nobre AC, Bloch G, Hyder F, Goldman-Rakic P, Shulman RG (1994): Functional magnetic resonance imaging of human prefrontal cortex activation during a spatial working memory task. Proc Natl Acad Sci USA 91:8690-8694

McCarthy G, Blamire AM, Rothman DL, Gruetter R, Shulman RG (1993): Echo-planar magnetic resonance imaging studies of frontal cortex activation during work generation in humans. Proc Natl Acad Sci 90:4952-4956

Ogawa S, Lee T-M, Nayak AS, Glynn I' (1990): Oxygenationsensitive contrast in magnetic resonance image of rodent brain at high magnetic fields. Mag Reson Med $14: 68-78$

Ogawa S, Menon RS, Tank DW, Kim SG, Merkle H, Ellermann JM, Ugurbil K (1993): Functional brain mapping by blood oxygenation level-dependent contrast magnetic resonance imaging. A comparison of signal characteristics with a biophysical model Biophys J 64:803-812

Ogawa S, Tank DW, Menon R, Ellermann JM, Kiom SG, Merkle H, Ugurbil K (1992): In rinsic signal changes accompanying sensory stimulation: functional brain mapping with magnetic resonance imaging. Proc Natl Acad Sci USA 89(13):5951-5955

Oldfield RC (1971): The assessment and analysis of handedness: the Edinburgh Inventory. Neuropsychologia 9:97113

Ramsey NF, Kirkby BS, Gelderen Pv, Berman KF, Duyn JH, Frank JA, Mattay VS, Horn JDv. Esposito G, Moonen CTW, Weinberger DR (1996a): Functional mapping of human sensorimotor cortex with 3D BOLD fMRI correlates with $\mathrm{H}_{2}{ }^{15} 0$ PET rCBF. J Cereb Blood Flow Metab 16:755-764

Ramsey NF, Tallent K, Gelderen PV, Frank JA, Moonen CTW, Weinberger DR (1996bi: Reproducibility of human 3D fMRI brain maps accuired during a motor task. Hum Brain Mapping 4(2):113-121

Renshaw PF, Yurgelun-Todd DA, Cohen BM (1994): Greater hemodynamic response to photic stimulation in schizophrenic patients: An echo planar MRI study. Am J Psychiatry 151:1493-1495

Rubin P, Holm S, Friberg L, Videbeck P, Andersen HS, Bendsen BB, Strosmo N, Larsen JK, Lassen NA, Hemmingsen $R$ (1991): Altered modulation of prefrontal and subcortical brain activity in newly diagnosed schizophrenia and schizophreniform disorder: A regional cerebral blood flow study. Arch Gen Psychiatry 48:987-995

Santha AKS, Ostuni JL, Mattay VS, Callicott JH, Frank JA, Weinberger DR (1997): Effectiveness and pitfalls of interpolation methods used in fMRI data analysis. International Society for Magnetic Resonance in Medicine, Vancouver, BC, Canada, 1914

Schroder J, Wenz F, Schad LR, Baudenistel K, Knopp MV (1995): Sensorimotor cortex and supplementary motor area changes in schizophrenia: A study with functional magnetic resonance imaging. Br J Psychiatry 167:197-201

Talairach J, Tournoux P (1993): Referentially oriented cerebral MRI anatomy. New York, Georg Thieme Verlag

Unser M, Aldroubi A, Gerfen CA (1993). A multiresolutional image registration procedure using spline pyramids. Proc. SPIE, San Diego, CA

Watson C (1991): Basic Human Neuroanatomy. Boston, Little, Brown, and Company

Van Gelderen P, Ramsey NF, Liu G, Duyn JH, Frank JA, Weinberger DR, Moonen CTW (1995): Three-dimensional functional magnetic resonance imaging of human brain on a clinical 1.5-T scanner. Proc Natl Acad Sci USA 92:6906-6910

Weinberger DR, Berman KF (1996): Prefrontal function in schizophrenia: Confounds and controversies. Philos Trans Royal Soc Med 351:1495-1503

Weinberger DR, Mattay V, Callicott J, Kotrla K, Santha A, Gelderen PV, Duyn J, Moonen C, Frank J (1996): FMRI application in schizophrenia research. Neuroimage 4:S118-S126

Weisskoff RM (1995): Functional MRI: are we moving towards artifactual conclusions? Or fMRI fact or fancy? NMR Biomed 8:101-103

Wenz F, Schad LR, Knopp MV, Baundendistel KT, Flomer F, Schroder J, Kaick Gv (1994): Functional magnetic resonance imaging at 1.5T: Activation pattern in schizophrenic patients receiving neuroleptic medication. Magn Reson Med 12:975-982

Wyatt R (1993): Practical psychiatric practice: clinical interview forms, rating scales, and practice handouts, Washington, DC, American Psychiatric Press

Yurgelun-Todd DA, Waternaux CM, Cohen BM, Gruber SA English CD, Renshaw PR (1996): Functional magnetic resonance imaging of schizophrenic patients and comparison subjects during word production. Am J Psychiatry 153:200-205 